\title{
Direct Numerical Simulation of a Turbulent Flow Using a Spectral/hp Element Method
}

\author{
Andrei Shishkin and Claus Wagner \\ DLR - Institute for Aerodynamics and Flow Technology \\ Bunsenstr.10, D-37073 Göttingen, Germany \\ e-mail: Andrei.Shishkin@dlr.de, Claus.Wagner@dlr.de
}

\section{Summary}

Direct numerical simulation (DNS) of incompressible turbulent pipe flow was carried out on unstructured grids for a Reynolds number based on the friction velocity and the pipe diameter of $R e_{\tau}=360$ using the spectral/hp element method (SEM) by Karniadakis and Sherwin [3]. The main objective was to investigate the computational aspects of this DNS with respect to accuracy, CPU time and memory requirements. DNS results of evaluated statistical moments of up to fourth order agree well with data from the literature. A conducted performance study reveals the computational requirements for DNS of turbulent flows using this SEM.

\section{Introduction}

Numerical simulations of turbulent flows by means of Direct Numerical Simulation (DNS) and Large Eddy Simulation (LES) have been the subject of intensive research during the last years. These techniques require accurate numerical methods which do not produce significant artificial viscosity. Spectral methods which provide accurate spatial discretization have been used successfully in the past mainly for simple geometries. In these methods the flow variables are expanded in terms of smooth (infinitely differentiable), mostly orthogonal trial functions, which are defined over the whole computational domain. Typical trial functions are the Fourier series components (domain with periodic boundary conditions) or Chebyshev and Legendre polynomials (nonperiodic boundary condition). Principally, DNS and LES of turbulent flows in complex geometries can be conducted using the spectral/hp element method (SEM) by Karniadakis and Sherwin [3]. In this method high-order polynomials are used to approximate the flow fields within finite elements to provide spectral accuracy on unstructured meshes. The advantage is that this SEM inherits the geometrical flexibility of finite element methods and the exponentially accurate polynomial resolution of spectral methods.

The main objective of this work is to use the SEM for a full 3D DNS of a turbulent flow in a computational domain with curved boundaries (which is 
a challenge for SEM) on an unstructured mesh and to compare the obtained results with those from the literature. The turbulent pipe flow was selected as a test case since it meets the above requirements although the geometry is fairly simple for the SEM. The other advantage is that turbulent pipe flows have been intensively investigated in numerical and experimental studies. Some of the results are collected in the AGARD Advisory report No. 345 [1, Ch.5].

The structure of the paper is the following. The computational domain and parameters are presented in section 2 . Section 3 contains a short description of the used SEM, i.e the $\mathcal{N} \varepsilon \kappa \boldsymbol{T} \boldsymbol{\alpha} \boldsymbol{r}$ code by Karniadakis and Sherwin [3]. In Section 4 CPU time and storage requirements of the SEM are compared with those of the second order accurate finite volume method (FVM) by Wagner and Friedrich [5]. In this comparison the requirements of FVM serve only as a reference since the method is only applicable in simple computational domains due to the use of Fourier transformations in streamwise and azimuthal directions. Finally, in Section 5 the mean streamwise velocity $\left\langle u_{z}\right\rangle$, the rms velocity fluctuations $u_{\alpha, r m s}$, the skewness $S_{\alpha}$ and flatness $F_{\alpha}$ factors $(\alpha=z, \varphi, r)$ are compared to turbulent pipe flow data by Loulou et al $[1,4]$ who used a B-spline spectral method (B-spline SM) for their DNS and by Wagner and Friedrich [5]. Further, experimental data from Laser Doppler Anemometry (LDA) and Particle Image Velocimetry (PIV) measurements by Durst et al [2] and by Westerweel et al [8], respectively, are included.

\section{Computational details}

We consider a turbulent pipe flow for a Reynolds number, based on friction velocity $\hat{u}_{\tau}$, pipe diameter $\hat{D}$ and kinematic viscosity $\hat{\nu}$, of $R e_{\tau}=\frac{\hat{u}_{\tau} \hat{D}}{\hat{\nu}}=360$, where (.) stands for dimensional quantities. The computational domain $\Omega$ is a pipe section of diameter $D$ and length $L=5 D$. For an incompressible Newtonian fluid the flow is governed by the dimensionless Navier-Stokes equations

$$
\frac{\partial \boldsymbol{u}}{\partial t}+(\boldsymbol{u} \cdot \nabla) \boldsymbol{u}=-\nabla p+\frac{1}{R e_{\tau}} \nabla^{2} \boldsymbol{u}+\boldsymbol{F}, \quad \nabla \cdot \boldsymbol{u}=0
$$

where $\boldsymbol{u}$ denotes the velocity vector field, $p$ - the pressure field, $\boldsymbol{F}$ - the forcing vector. For a fully developed turbulent pipe flow the driving force $\boldsymbol{F}$ corresponds to the mean pressure gradient in streamwise $z$-direction, $\left\langle\frac{\partial p}{\partial z}\right\rangle=-4$. At the solid wall $\left.\mathbf{u}\right|_{\partial \Omega}=0$ enforces the impermeability and no-slip boundary conditions. Furthermore, periodical boundary conditions are applied in streamwise direction. The simulations were started from an initial turbulent field which was obtained in a DNS using a second order accurate FVM. 


\section{Spectral/hp element method for incompressible flows}

The $\mathcal{N} \varepsilon \kappa \mathcal{T} \boldsymbol{\alpha} \boldsymbol{r}$ code exploits structured and unstructured conforming meshes or a combination of both. Considering the generation of 3D meshes, this admits any combination of hexagonal, tetrahedral and prismatic elements provided that the corresponding faces of contiguous elements coincide.

The velocity and pressure fields are sought in terms of series expansions. The velocity field, for example, is expressed as

$$
u(\boldsymbol{x}, t)=\sum_{i} \hat{u}_{i}(t) \varphi_{i}(\boldsymbol{x}),
$$

where $\varphi_{i}(\boldsymbol{x})$ denotes an $i$-th mode (base function) and $\hat{u}_{i}(t)$ - the corresponding expansion coefficient. Each mode $\varphi_{i}(\boldsymbol{x})$ is a piecewise polynomial function supported by one element of the mesh. The system $\left\{\varphi_{i}(\boldsymbol{x})\right\}$ is constructed using the tensor product of the polynomials, orthogonal over a simplex, and the classical Jacobi polynomials (for details see [3, Ch.3]).

The solution of Eq. (1) is obtained using the splitting method by Karniadakis and Sherwin [3, p.247] which combines semi-implicit time integration using a forward Adams scheme $[3, \mathrm{p} .193]$ with the enforcement of the continuity equation. This leads to a Poisson equation for the pressure field and a Helmholtz equation for the velocity field which are solved with a preconditioned conjugate gradient method using the Schur complement (static condensation) technique.

\section{Performance study}

We construct an unstructured mesh consisting of 79860 prisms presented in Fig. 1. The elements are uniformly distributed along the axis and nonuniformly along the pipe radius according to a quadratic law to provide higher spatial resolution in the vicinity of the wall. The highest order of the expansion polynomials is taken equal to 3 for both components of the tensor product, resulting in 40 modes within each element.

In the spectral/hp element approach the combination of two parameters, i.e. the characteristic size $h$ of the elements and the order $p$ of the polynomial modes, defines the mesh resolution. In order to compare the spatial resolution achieved in SEM and FVM simulations it seems reasonable to aggregate both $h$ and $p$ characteristics as follows. Consider the number $M_{d f}$ of degrees of freedom of the expansions (2), i.e. the number of independent global expansion modes, to calculate the average value $M_{d f / e l}(e)$ of degrees of freedom for each element $e$. The ratio $\delta(e)=\frac{M_{d f / e l}(e)}{v(e)}$, where $v(e)$ is the volume of the element $e$, serves as a measure of the spatial resolution of the element $e$. Finally, we define a piecewise constant function $\delta(x)=\delta(e), \boldsymbol{x} \in e$, for each point $\boldsymbol{x}$ of the computational domain $\Omega$, which indicates the quality of 
spatial resolution. Considering a cylindrical domain, the averaged functions $\delta(r, \varphi)=\frac{1}{L} \int \delta(\boldsymbol{x}) d z$ and $\delta(r)=\frac{1}{2 \pi L} \iint \delta(\boldsymbol{x}) d z d \varphi$, provide the appropriate spatial resolution characteristics of the mesh.

The well resolved DNS by Wagner and Friedrich [5] who used a nonequidistant cylindrical structured grid with $N_{z}=256, N_{\varphi}=128, N_{r}=70$ grid points in $z, \varphi$ and $r$-directions, respectively, serves as reference case. For this mesh and the FVM the function $\delta(r, \varphi)$ can be determined taking $M_{d f / e l}=1$.

In Fig. 2 profiles of the mesh resolution functions $\delta(r, \varphi)$ of the SEM and the above mentioned FVM are presented. It is observed that the FVM mesh has a significantly higher resolution near the centerline due to its singularity at the axis. The unstructured mesh has a comparable resolution beyond the center zone and even higher resolution than the FVM mesh in the viscous sublayer near the rigid wall. Therefore, it is concluded that the constructed SEM mesh is fine enough to resolve all relevant turbulent scales.

The spectral element simulations were conducted in a parallel mode using 10 processors of the 32 -bit Linux Cluster machine. The main characteristics of the meshes used and the computational requirements are summarized in Table 1. It is observed that the SEM needs 80 times more CPU time and 60 times more Random Access Memory (RAM) than the FVM. It must be noted that in a similar performance study the second order accurate FVM for curvilinear staggered grids by Wesseling et al [7] required 358 times more CPU time and 32 times more RAM than the FVM for structured cylindrical grid (see Wagner and Dallmann[6]).

\section{$5 \quad$ Numerical results}

In Fig. 3-6 the turbulence statistics obtained in the DNS with the SEM (SEM DNS) are compared with results of different authors.

In Fig. 3 profiles of the mean axial velocity obtained in the SEM DNS are depicted together with those obtained in the FVM DNS by Wagner and Friedrich [5], in the B-spline SM DNS by Loulou et al [4] and in the PIV measurements by Westerweel et al [8]. The comparison reveals a good agreement to the reference data. Fig. 4 displays the root-mean-square (rms) values of the fluctuating velocity components for the SEM DNS in comparison with the FVM DNS by Wagner and Friedrich [5], the B-spline SM DNS by Loulou et al [4], the PIV measurements by Westerweel et al [8] and the LDA measurements by Durst et al [2]. Again, an exellent agreement of all the data is observed. The skewness and the flatness factors (Figures 6,5) are compared with the numerical data by Wagner and Friedrich [5] and by Loulou et al [4] and the measurements by Durst et al [2]. There is a certain variance in the behavior of the skewness $S_{r}$ and the flatness $F_{r}$ factors of the radial velocity component. Contrary to the low-order FVM DNS, the skewness factors $S_{r}$ of the SEM DNS and the B-spline SM DNS change their sign near the wall in an 
agreement with the measurements. Fig. 6 exhibits a discrepancy in the flatness factor $F_{r}$ obtained in the numerical simulations and in the experiments. The measurements provide lower values of $F_{r}$ than the simulations near the wall. Presently this contradiction is an open problem and needs to be investigated precisely in the future. We note also that the SEM DNS data present a lower near-wall value of $F_{r}$ than other considered numerical methods.

\section{Conclusion}

In the paper we investigated the computational aspects of DNS of a turbulent pipe flow using the spectral/hp element method. To this end, we generated an unstructured mesh consisting of 79860 prisms and conducted the simulation using the SEM code $\mathcal{N} \varepsilon \boldsymbol{\kappa} \boldsymbol{T} \boldsymbol{\alpha r}[3]$.

The statistical results obtained in the SEM DNS are in a good agreement with other numerical and experimental data. Particularly, the skewness and the flatness factors of SEM DNS agree better with the experimental data than those of FVM DNS. As compared with the FVM for simple geometries the SEM needs substantially more CPU time and RAM. Note that the considered version of SEM does not exploit the symmetry properties of the computational domain. Therefore, the SEM can be used for turbulent flow simulations in complex geometries with about the same computational expenses.

\section{Acknowledgments}

We are grateful to Dr. S.Sherwin for providing the $3 \mathrm{D}$ version of $\mathcal{N} \varepsilon \kappa \mathcal{T} \boldsymbol{\alpha r}$.

\section{References}

[1] AGARD Advisory Report No. 345. "A Selection of Test Cases for the Validation of Large-Eddy Simulations of Turbulent Flows", 1998

[2] F. Durst, J. Jovanovic, J. Sender. "Detailed measurements of the near wall region of turbulent pipe flows". Proc. 9th Symp. on Turbulent Shear Flows, Kyoto, Japan, August 16-18, 2/2/1-2/2/6 (1993)

[3] G.E. Karniadakis and S.J. Sherwin. "Spectral/hp Element Method for CFD". Oxford University Press, 1999.

[4] P. Loulou, R. Moser, N. Mansour, B. Cantwell. "Direct simulation of incompressible pipe flow using a b-spline spectral method". Technical Report TM 110436, NASA, 1997.

[5] C. Wagner, R. Friedrich. "On the turbulence structure in solid and permeable pipes". International Journal of Heat and Fluid Flow, 19(1998), 459-469.

[6] C. Wagner, U. Ch. Dallmann. "A direct Navier-Stokes solver for turbulent flows over round steps", in: W. Nitsche, R. Hilbig (Eds.), 1999, New Results in Numerical and Experimental Fluid Mechanics, Contributions to the 11th AG-STAB/DGLR Symposium, Berlin, Germany, 1998, Vieweg-Verlag 
[7] P, Wesseling, A. Segal, J.J.I.M. van Kan, C.W. Oosterlee, C.G.M. Kassels. "Finite volume discretization of the incompressible Navier-Stokes equations in general coordinates on staggered grids". Comp. Fluid Dynamics Journal, 16(1992), 27-33

[8] J. Westerweel, R.J. Adrian, J.G.M. Eggels, F.T.M. Nieuwstadt. "Measurements with particle image velocimetry on fully developed turbulent pipe flow at low Reynolds number". Proc. of the 6th Int. Symp. on Applications of Laser Tech. to Fluid Mechanics, Lisbon, Portugal, July 20-23, 1993

Table 1 Computational requirements of a spectral/hp element method (SEM) and a finite volume method (FVM) for structured cylindrical grid in DNS of turbulent pipe flow.

\begin{tabular}{|l|c|c|c|c|c|c|c|}
\hline & $N_{e l}$ & $M_{d f}$ & $\begin{array}{c}\text { number } \\
\text { of proc. }\end{array}$ & $\begin{array}{c}\text { memory } \\
\text { usage }\end{array}$ & $\begin{array}{c}\text { memory } \\
N_{e l}\end{array}$ & $\begin{array}{c}\text { memory } / \\
M_{d f}\end{array}$ & $\begin{array}{c}\text { CPU-time } / M_{d f} \\
\text { per time step }\end{array}$ \\
\hline SEM & 79860 & 1045770 & 10 & $8030 \mathrm{Mb}$ & $103 \mathrm{~Kb}$ & $7.86 \mathrm{~Kb}$ & $9.8 \cdot 10^{-4} \mathrm{sec}$ \\
FVM & 2293760 & 2293760 & 1 & $131.25 \mathrm{Mb}$ & 60 byte & 60 byte & $1.22 \cdot 10^{-5} \mathrm{sec}$ \\
\hline
\end{tabular}

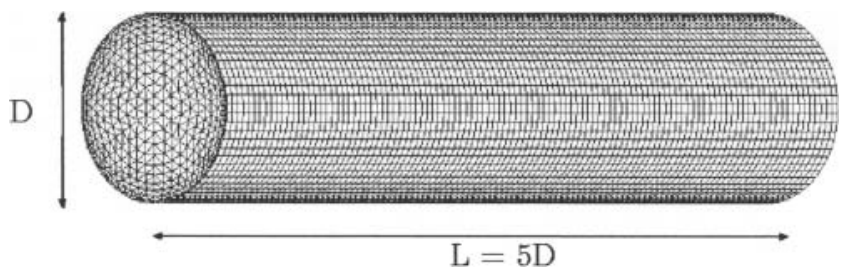

Figure 1 Unstructured mesh for the spectral/hp element method.

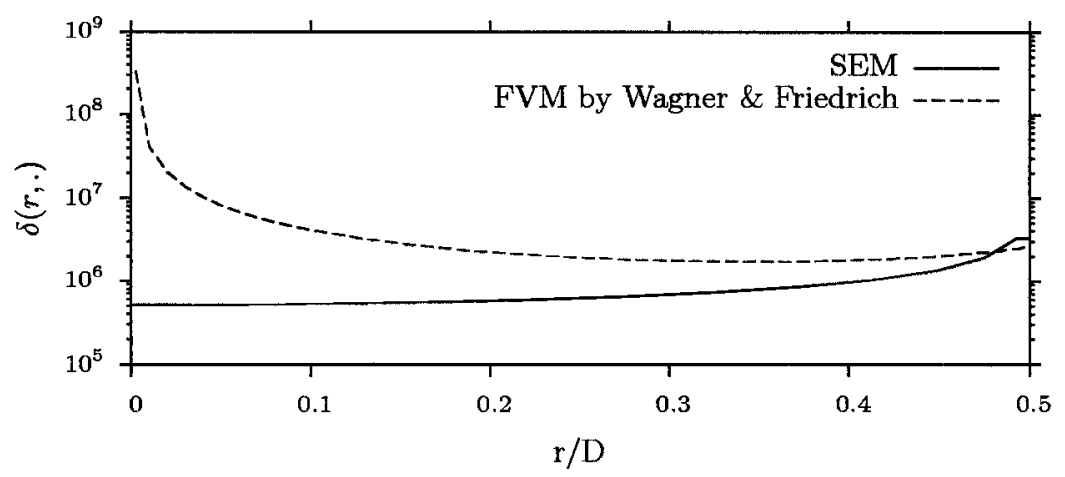

Figure 2 The profiles of the mesh resolution function $\delta(r, \varphi)$ (see Section 4) for the spectral/hp mesh (SEM) and the structured cylindrical non-equidistant grid $\left(N_{z}=256, N_{\varphi}=128, N_{r}=70\right)$ (Wagner and Friedrich [5] ). 


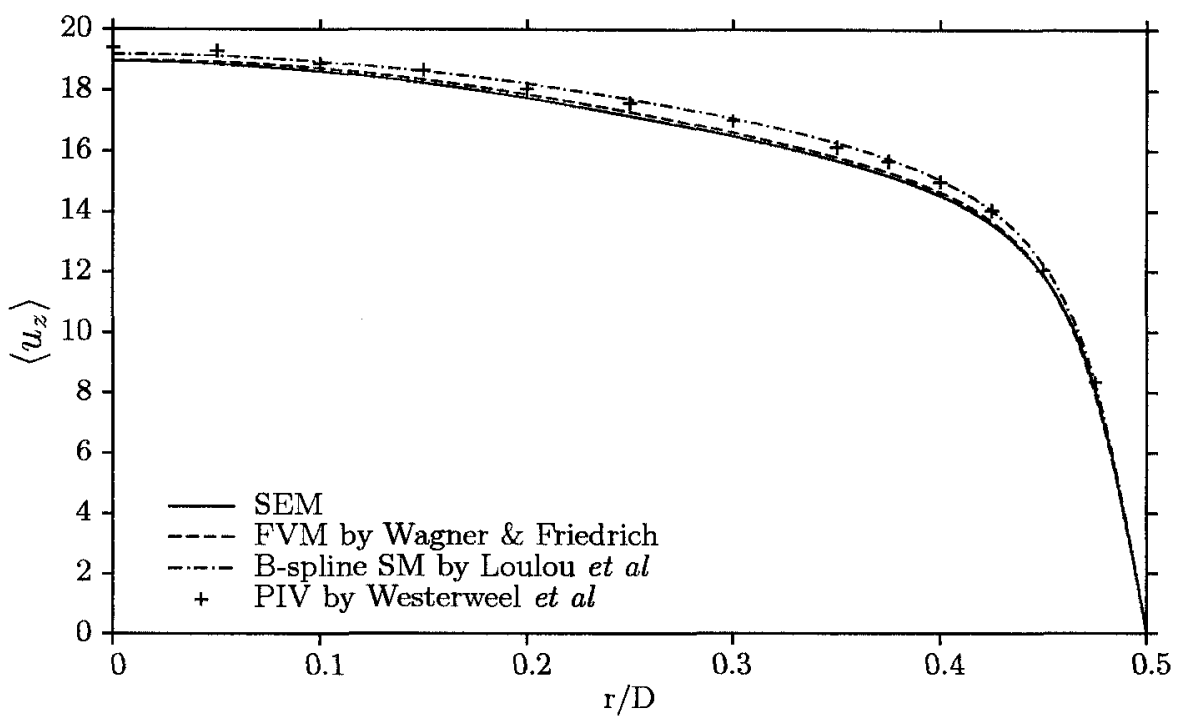

Figure 3 Mean streamwise velocity $\left\langle\bar{u}_{z}\right\rangle$ profiles obtained in the DNS of turbulent pipe flow using the spectral/hp element method, the FVM DNS by Wagner and Friedrich [5], the B-spline SM DNS by Loulou et al [4] and the PIV measurements by Westerweel et al [8].

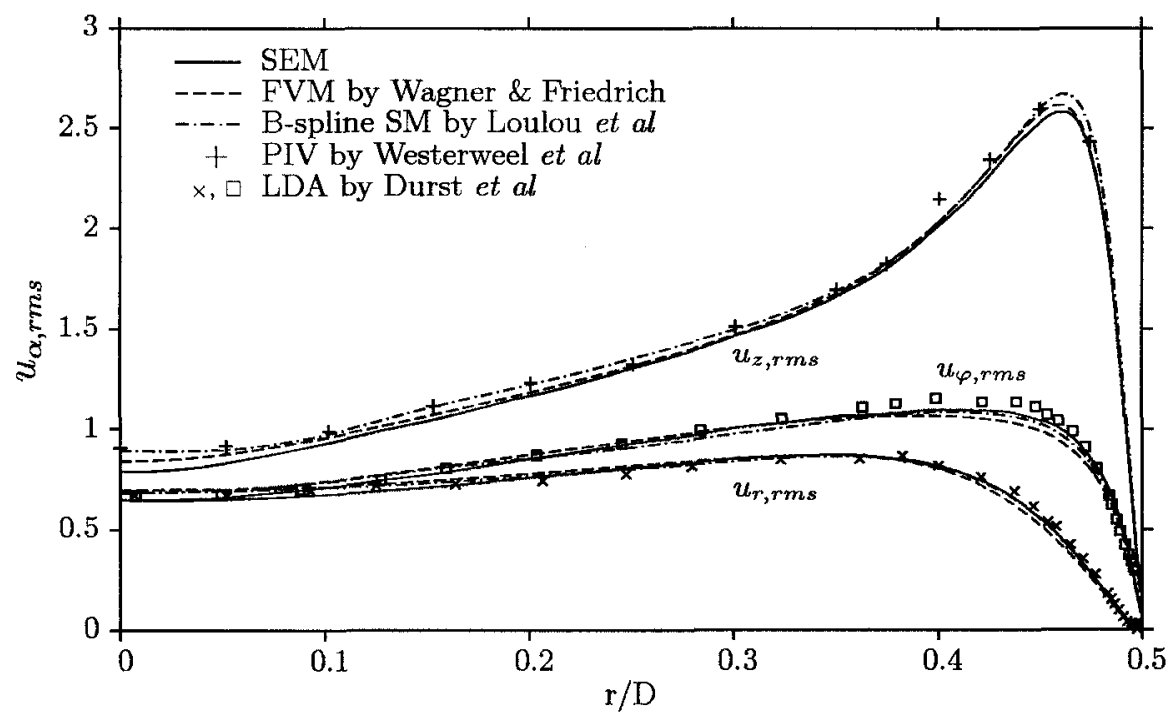

Figure 4 rms-velocities obtained in the DNS of turbulent pipe flow using the spectral element method, the FVM DNS by Wagner and Friedrich [5], the B-spline SM DNS by Loulon et al [4], the PIV measurements by Westerweel et al [8] and the LDA measurements by Durst et al [2]. 


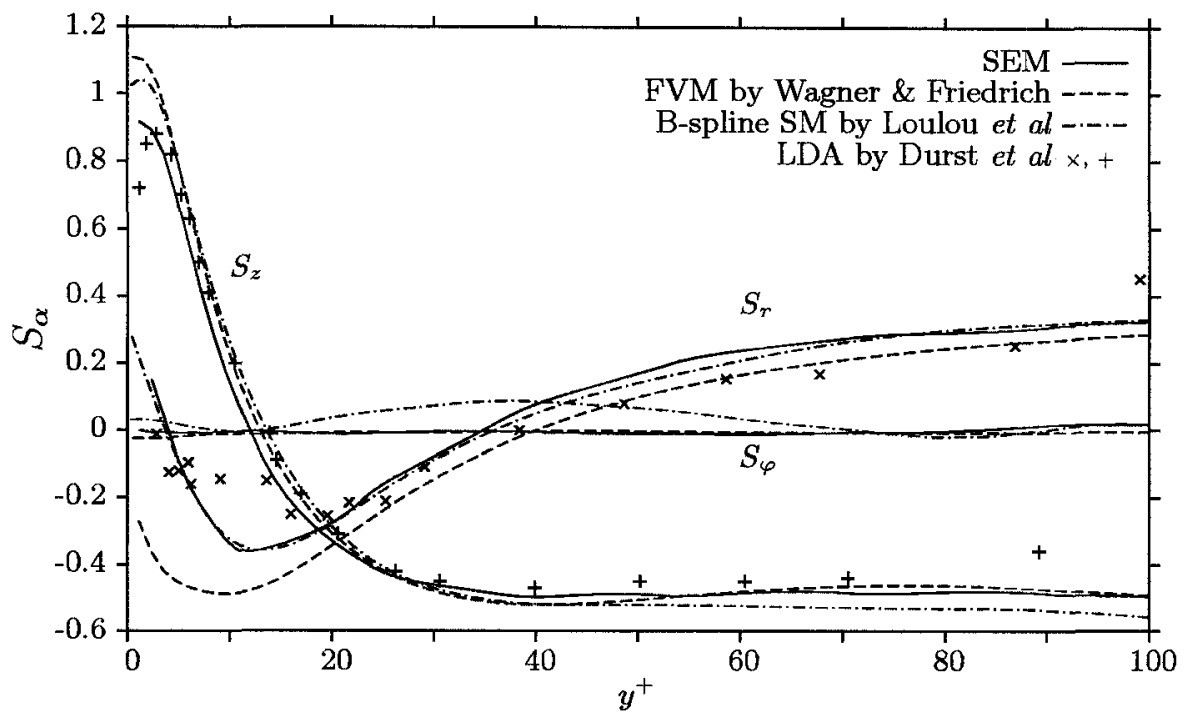

Figure 5 Skewness factors of the velocity fluctuations obtained in the DNS of turbulent pipe flow using the spectral element method, the FVM DNS by Wagner and Friedrich [5], the B-spline SM DNS by Loulou et al [4] and the LDA measurements by Durst et al [2].

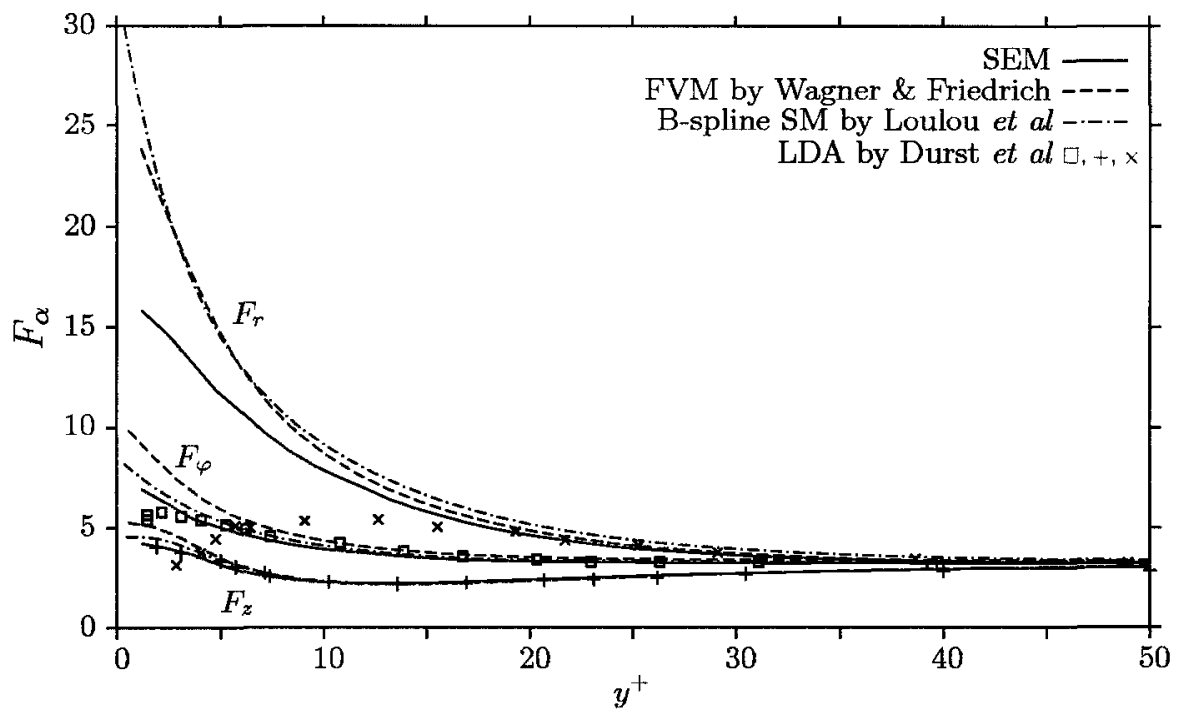

Figure 6 Flatness factors of the velocity fluctuations obtained in the DNS of turbulent pipe flow using the spectral element method, the FVM DNS by Wagner and Friedrich [5], the B-spline SM DNS by Loulou et al [4] and the LDA measurements by Durst et al [2]. 